\title{
Trastorno límite de la personalidad y el abuso sexual infantil
}

\author{
Borderline Personality Disorder and Child Sexual Abuse
}

\author{
Jeimy L. Pérez-Rivera ${ }^{a}$, Ana C. Pimentel-García ${ }^{b}$, Liana C. Hernández Díaz ${ }^{c}$, Mercedes S. \\ Martínez-Arce ${ }^{d}$, Gelacio Guzmán-Díaz ${ }^{e}$, Jesús Cisneros-Herrera ${ }^{f}$
}

\begin{abstract}
:
In this paper, relationship between sexual abuse in childhood as risk factor and the emergence of borderline personality disorder from a psychoanalytic view. Borderline personality disorder (BPD) is a pathology characterized by several symptoms, such as affective instability, feelings of emptiness, psychotic episodes, dichotomous thinking, cognitive distortions, impulsivity, fear of abandonment and self-harm behaviors. These symptoms, from a psychoanalytic view, may be manifestations of a psychic imbalance, which are a result of defense mechanisms that predominate in patients with BD. The theoretical proposal of Melanie Klein regarding the two positions (schizoid-paranoid and depressive) is especially relevant to understand intrapsychic dynamics of individuals who suffer from this disorder. Sexual abuse in childhood by itself is not enough to a BDP to appear; however, it is an experience that requires to rearrange psychic structure when there is no external support from family.
\end{abstract}

Keywords:

Borderline personality disorder, Sexual abuse in childhood, Psychoanalysis, Defence mechanisms

Resumen:

En el presente escrito se describe la relación entre el abuso sexual infantil y el desarrollo de trastorno límite de la personalidad desde la perspectiva psicoanalítica. El trastorno límite de la personalidad (TLP) es una patología caracterizada por diversos síntomas, entre ellos, inestabilidad afectiva, sentimientos de vacío, episodios psicóticos, pensamientos dicotómicos, distorsiones cognitivas, episodios de impulsividad, miedo al abandono y conductas auto lesivas. Dichos síntomas, desde la perspectiva psicoanalítica, pueden ser manifestaciones desarrolladas a partir del desequilibrio psíquico, derivado de la presencia de mecanismos de defensa cuya predominancia se evidencia en pacientes con TLP. La propuesta teórica de Melanie Klein acerca de las dos posiciones (esquizoparanoide y depresiva) es de especial relevancia para entender la dinámica intrapsíquica de las personas que padecen este tra storno. El abuso sexual infantil por sí mismo no es suficiente para la génesis del TLP, sin embargo, es una experiencia que demanda reacomodos en la estructura psíquica a falta del apoyo externo de la familia.

Palabras Clave:

Trastorno límite de la personalidad, Abuso sexual infantil, Psicoanálisis, Mécanismos de defensa

\section{Trastorno límite de la personalidad}

En los últimos años, un tema de creciente interés ha sido el estudio de la relación entre la adversidad temprana y las dificultades psicológicas en fases posteriores de la vida partiendo de la idea de que la naturaleza transaccional persona-contexto está en el origen de los problemas psicológicos. Aunque diferentes experiencias tempranas pueden desempeñar un papel importante en la vida adulta, se ha investigado especialmente la relación entre trauma infantil y diferentes trastornos psicológicos en la adultez. Así, el trauma infantil se ha asociado con la aparición de gran variedad de alteraciones psicopatológicas, entre ellas el trastorno límite de la personalidad (1).

\footnotetext{
${ }^{a}$ Autor de Correspondencia, Universidad Autónoma del Estado de Hidalgo, https://orcid.org/0000-0002-3600-0606, Email: pe362345@uaeh.edu.mx ${ }^{b}$ Universidad Autónoma del Estado de Hidalgo, https://orcid.org/0000-0002-1175-2617, Email: pi354857@uaeh.edu.mx c Universidad Autónoma del Estado de Hidalgo, https://orcid.org/0000-0002-2574-2006, Email: he362913@uaeh.edu.mx ${ }^{d}$ Universidad Autónoma del Estado de Hidalgo, https://orcid.org/0000-0002-8771-2857, Email: ma358898@uaeh.edu.mx ${ }^{e}$ Universidad Autónoma del Estado de Hidalgo, https://orcid.org/0000-0003-4869-8658, Email: gelacio_guzman@uaeh.edu.mx ${ }^{\mathrm{f}}$ Universidad Autónoma del Estado de Hidalgo, https://orcid.org/0000-0003-1370-8086, Email: jesus_cisneros@uaeh.edu.mx
} 
El trastorno límite de la personalidad (TLP) está comprendido dentro de los trastornos de personalidad grupo B según la clasificación del DSM-5 (2), y se le considera un trastorno psiquiátrico grave. Este trastorno es heterogéneo -pues se presenta de manera muy diferente en cada paciente-, complicado de delimitar, con presentaciones clínicas y conductuales con mucha variabilidad interindividual e intraindividual, es decir, también varía en un mismo individuo a lo largo de su vida. Sin embargo, como un rasgo general, Frías y cols. (3) mencionan que las personas con TLP presentan una falta de autenticidad y de sentimiento de identidad (o difusión de la identidad). Estos los síntomas básicos funcionan como una defensa que les ayuda a afrontar la vida de una manera más operativa.

Estos sujetos pueden tener el mismo tipo de sentimientos y percepciones que todos, pero viven y sienten de una forma más intensa, también son más vulnerables porque adquieren cualidades desproporcionadas. Por ello, se debe de tener en cuenta los criterios diagnósticos del Manual diagnóstico y estadístico de los trastornos mentales DSM-5 (2), de los cuales deben manifestar cinco (o más) de los siguientes criterios:

Trastorno límite de personalidad 301.83 (F60.3)

- Esfuerzos desesperados para evitar el desamparo real o imaginado.

- Patrón de relaciones interpersonales inestables e intensas que se caracteriza por una alternancia entre los extremos de idealización y de devaluación.

- $\quad$ Alteración de la identidad: inestabilidad intensa y persistente de la autoimagen y del sentido del yo.

- Impulsividad en dos o más áreas que son potencialmente autolesivas (p. ej., gastos, sexo, drogas, conducción temeraria, atracones alimentarios). (Nota: No incluir el comportamiento suicida ni las conductas autolesivas que figuran en el Criterio 5.)

- Comportamiento, actitud o amenazas recurrentes de suicidio, o conductas autolesivas.

- Inestabilidad afectiva debida a una reactividad notable del estado de ánimo (p. ej., episodios intensos de disforia, irritabilidad o ansiedad que generalmente duran unas horas y, rara vez, más de unos días).

- Sensación crónica de vacío.

- Enfado inapropiado e intenso, o dificultad para controlar la ira (p. ej., exhibición frecuente de genio, enfado constante, peleas físicas recurrentes).

- Ideas paranoides transitorias relacionadas con el estrés o síntomas disociativos graves
Entre las causas del desarrollo del TLP pueden describirse las experiencias de adversidades a edades tempranas (p. ej.: el abuso sexual), o crecer en un entorno disfuncional: un estilo de crianza parental con ausencia de límites, poco consistente o negligente a nivel emocional (4).

Los factores de desarrollo que convergen en las personas con TLP son la falta de estándares establecidos del bien y el mal durante la infancia y adolescencia, la ausencia de gozo en las relaciones con sus compañeros, y principalmente la reaparición de los síntomas que se suponían superados (5). Desde el punto de vista del desarrollo se puede considerar que los pacientes reviven una crisis infantil temprana en la cual temían que los intentos de separación de sus madres provocarían la desaparición de éstas y el abandono. En la forma adulta de esta crisis infantil, los individuos son incapaces de tolerar períodos de soledad y el temor de que los seres más significativos los abandonen. Las razones de esta fijación, según Kernberg (6), están relacionadas con una alteración de la disposición emocional de la madre durante este período crítico, debido al exceso constitucional de agresión en el niño o a problemas de la función materna, o a una combinación de ambas. En el niño aparecen celos, frustraciones y envidias al mismo tiempo actúa de manera grosera ante sus padres hasta imaginarlos destruidos.

\section{Psicoanálisis y Mecanismos de defensa en el TLP}

Los mecanismos de defensa utilizados en el TLP son, sobre todo, de carácter primitivo y producen resultados patológicos al ser éstos usados en exceso, con intensidad exagerada o de manera aislada. Estos mecanismos de defensa primitivos son: la escisión, la idealización primitiva, la identificación proyectiva, la omnipotencia, la devaluación y la negación, y proceden de etapas muy precoces del desarrollo evolutivo (6).

En primer lugar, con respecto a la escisión, que origina y subyace en todas las demás defensas, generadas en la principal operación defensiva en este tipo de pacientes. La causa fundamental para la utilización masiva de este mecanismo es la debilidad yoica. La escisión consiste en separación de un mismo objeto en dos polaridades, una buena, amada, que brinda satisfacción y placer, y otra mala, odiada, que produce frustración y dolor. En el mundo interno, el objeto externo da lugar a dos objetos internos ubicados en estos dos polos, con los cuales el yo se relaciona de acuerdo con las características que les atribuye (7).

En segundo lugar, con respecto a las idealizaciones primitivas, son un mecanismo de defensa contra el objeto malo. Cuanta mayor angustia provoca el objeto malo, mayor es la necesidad de idealizar a objeto bueno y sentir 
su protección (7). En la etapa adulta, la idealización es patológica, porque crea confusión entre lo idealizado y la realidad, este mecanismo es empleado de una forma masiva, repetitiva, indiferenciada y primitiva en el TLP. Se trata de una idealización casi psicótica, total, con falta de sentido de realidad en algunas ocasiones. Los objetos escindidos y proyectados (en bueno y malo) son totalmente buenos o malos, por efecto de la escisión y también de la idealización. Ni las devaluaciones ni las idealizaciones son fijas, sino que en unos segundos pueden cambiar en una misma persona, de sentirse perfecto a sentirse un mediocre varias veces al día. Debido a la intensa vulnerabilidad que activa los mecanismos de defensa, todo adquiere unas características desproporcionadas.

En tercer lugar, se encuentra la identificación proyectiva, mecanismo mediante el cual el yo expulsa de sí los elementos intolerables de su experiencia y los deposita en el objeto externo (7). Las consecuencias del uso masivo de este mecanismo conducen a un empobrecimiento yoico e incapacidad de discriminar entre sujeto y objeto, en un estado de confusión, perturbaciones en el sentido de identidad. No solo lanza al exterior sus propias emociones, sino también partes de su propio yo, capaces de hacerse cargo de la realidad psíquica. La causa desencadenante de la utilización de este mecanismo es la intolerancia a la separación, la necesidad de control omnipotente, la envidia, los celos, la desconfianza y la ansiedad persecutoria.

En cuarto lugar, hace referencia a los mecanismos defensivos de omnipotencia y devaluación, la utilización de estas defensas constituyen un recurso maníaco ante sentimientos depresivos, de devaluación, ruina y vacío. La omnipotencia es una fantasía de control sobre los objetos que se acompaña de la devaluación de estos. Por último, con respecto al acting out como mecanismo defensivo, realiza una utilización masiva y repetida de hetero y/o autoagresividad, por lo que las causas eran, como vimos en líneas anteriores, la angustia de fragmentación, la inestabilidad yoica, los afectos depresivos que amenazan con desbordarla de forma constante y la necesidad de confusión y de negar la realidad por falta de representaciones internalizadas (8).

\section{Abuso sexual infantil}

Hughes y cols. (9) concluyen que a pesar de que un gran número de personas con TLP refieren una historia de negligencia o abuso en la infancia, no está claro que el abuso sea un antecedente necesario para desarrollar posteriormente TLP, aunque sí que es una clara amenaza, en caso de negligencia o abuso, donde el desarrollo "normal" se vea amenazado.
El Sistema para el Desarrollo Integral de la Familia (10) menciona que el abuso sexual infantil (ASI) es el acto que comete una persona de la misma o mayor edad, que involucra al menor a realizar actividades sexuales (caricias, besos o tocamientos; a ver y escuchar pornografía o exhibir los genitales), las cuales no comprende totalmente. Por lo general, el ASI es perpetuado por una persona dentro del núcleo familiar o allegada a la familia de la víctima.

Por su parte, Revello (11) menciona que la experiencia de ASI, produce ajenidad, esa partición del yo que acontece con la experiencia traumática, queda por fuera del sujeto y es vivenciada como ajena, como un imposible. En tanto la vivencia, es generalmente reprimida, encerrada, enterrada, como lo que no puede haber sido, como aquello que no es posible representar. De esta manera se explica el desarrollo de los trastornos en el pensamiento debido a que el acto es difícil de representar. Pifarré (12) menciona que los trastornos del pensamiento se han dividido en trastornos del curso y trastornos del contenido del pensamiento, los trastornos más comunes en victimas de ASI son: 1) Pensamiento circunstancial; la información dada es excesiva, redundante, existe dificultad para seleccionar las ideas y no puede discernirse entre aquello que es esencial y aquello que es accesorio, con la misma importancia. 2) Disgregación; existe una pérdida de la idea directriz como consecuencia de la rotura de las asociaciones normales, fluyen los pensamientos sin conexión lógica. 3) Bloqueo del pensamiento: es la interrupción súbita del curso del pensamiento, antes de completar una idea, sin que exista razón alguna aparente que lo explique, al menos para el observador externo.

Sin embargo, Segal (13) menciona que la reparación propiamente puede considerarse una defensa, en víctimas de ASI, ya que se ocupa activamente tanto de controlar la destructividad como de reparar y restaurar el daño realizado, sin que aparezcan sentimientos de culpa debido a lo acontecido.

De ese modo, los mecanismos de defensa y afrontamiento son activados por una baja tolerancia a la angustia, frustración y depresión, de esta manera recurre a defensas primitivas como: la identificación proyectiva, escisión, constricción, a la fantasía, negación, idealización primitiva, control omnipotente y regresión en la forma de evaluación (5).

Así mismo Kemberg y cols. (5) mencionan que las funciones específicas impactan en la atención, orientación espacial y memoria de los niños en cuanto a las funciones no específicas crean un sentido de conciencia frágil, por un déficit yoico, como es la falta de control de impulsos, poca tolerancia a la frustración, bajo nivel de tolerancia a la angustia, baja tolerancia a la 
depresión, por lo el niño no es capaz de mostrar todo su potencial académico.

Sin embargo, Pereda (14) en su revisión bibliográfica, confirma la relación entre ASI y el desarrollo posterior de varios tipos de problemas, entre los que se destaca el TLP (entendido como problema emocional).

\section{Conclusión}

Los mecanismos de defensa identificados en el TLP corresponden a las fases tempranas de la vida del niño. En ese momento son mecanismos normales e inevitables para la estructuración del aparato psíquico, pero su predominancia en la etapa adulta resulta patológico. Para el recién nacido, la tarea principal es dominar la angustia persecutoria ocasionada por la presencia del objeto malo. Esta tarea es más fácil o difícil de acuerdo con las condiciones reales de la vida del niño. Cuando la frustración, el displacer y el dolor predominan sobre la satisfacción y el placer, la angustia persecutoria se vuelve incontrolable, por lo que entran en juego estos mecanismos primitivos de defensa de manera masiva. Sólo si las experiencias de satisfacción predominan sobre las de frustración, puede formarse un yo que pueda prescindir de esos mecanismos primitivos.

Los autores revisados en el presente trabajo muestran que el haber sufrido ASI constituye un factor de riesgo en el posterior desarrollo en la edad adulta de TLP, aunque no necesariamente debe considerarse una causa primaria ya que las historias ASI tienden a relacionarse con otros factores de riesgo para TLP. Esto influye que estas vivencias de ASI, en edades tempranas puedan ser olvidadas y no afecten en tanta medida en el posterior desarrollo del TLP.

El abuso sexual es una experiencia que perturba el equilibrio psíquico, pero que por sí mismo no necesariamente provoca el TLP. Es la experiencia repetida de frustraciones que impiden la sustitución de los mecanismos primitivos por otros más sofisticados. Su asociación con el abuso sexual infantil proviene del hecho de que este abuso en muchos casos es constante a lo largo de años. En casos en que el abuso es una experiencia única, pero se inserta en una cadena más generalizada de frustraciones y agresiones, el TLP es más probable.
[2]Asociación Americana de Psiquiatría. Manual diagnóstico y estadístico de los trastornos mentales. 5ta ed. Buenos Aires, Bogotá, Caracas, Madrid, México, Porto Alegre: Medica Panamericana. 2013.

[3] Frías Á, Palma C, Farriols N, González L. Sexuality related issues in borderline personality disorder: A comprehensive review. Personality and mental health, 2016; 10: 216-231. doi: 10.1002/pmh.1330.

[4] Levy K N. The implications of attachment theory and research for understanding borderline personality disorder. Development and Psychopathology. 2005; 17(4): 959-986. doi: $10.1017 /$ S0954579405050455.

[5] Kernberg P, Weiner A, Bardenstein K. Organización limítrofe de la personalidad. En Paula K (Eds.), Trastornos de la personalidad en niños y adolescentes. Mexico: Manual Moderno. 2002: 86-97.

[6] Kernberg O. Desórdenes fronterizos y narcisismo patológico. Buenos Aires: Ed. Paidós; 1975.

[7] Klein M. Notas sobre algunos mecanismos esquizo-paranoides. En Desarrollos en psicoanálisis. Buenos Aires: Hormé; 1946.

[8] Monzón S, Fernández M. Guía diagnóstica para el Trastorno Límite de la Personalidad en adolescentes. Psicopatol. Salud Mental. Junio 2016; 29(1): 55-65

[9] Hughes A, Crowell S, Uyeji L. \& Coan J. A developmental neuroscience of borderline pathology: emotion dysregulation and social baseline theory. Journal of Abnormal Child Psychology. 2012; 40(1): 21-33.

[10] Sistema Nacional DIF. Re: ¿Sabes que es el abuso sexual a niñas y niños? [Mensaje en un blog]. 23 de noviembre de 2017. Recuperado de: https://www.gob.mx/difnacional/articulos/sabes-que-es-el-abuso-sexual-aninas-y-ninos?idiom $=e s$

[11] Revello M. La atemporalidad del trauma en víctimas de abuso sexual infantil: su incidencia en la construcción el psiquismo y de los registros corporales (Trabajo final de grado, Uruguay Universidad de la República Facultad de Psicología); 2015. Recuperado de: https://sifp.psico.edu.uy/sites/default/files/Trabajos\%20finales/\%20Arc hivos/tfg articulo de revision $0 . p d f$

[12] Pifarré J. Trastornos del pensamiento y del lenguaje. En Vallejo J. (Eds.), Introducción a la psicopatología y la psiquiatría. Madrid: Elsevier Masson; 2015: 699-709.

[13] Segal H. Introducción a la obra de Melanie Klein. Buenos Aires, Barcelona: Paidós; 2003.

[14] Pereda N, Gallardo D, Jiménez, P. Trastornos de personalidad en víctimas de abuso sexual infantil. Actas Españolas de Psiquiatría. Marzo-abril, 2011; 39(2): 131-136.

\section{Referencias}

[1] Janssen I, Krabbendam L, Bak M, Hanssen M, Vollebergh W, Graaf R, Os J. Childhood abuse as a risk factor for psychotic experiences. Acta Psychiatrica Scandi. 2004; 109(1): 38-45. 